\section{Compact tunable lowpass filter with sharp roll-off and low insertion loss}

T. Zhang1, Z.-Q. Cai ${ }^{1}$, Y. Yang ${ }^{2}$, J.-F. Bao ${ }^{1}$ and Y.-W. Wang $^{3}$

A novel continuously tunable lowpass filter (LPF) with compact size, sharp roll-off and low insertion loss is presented in this paper. The filter employs two varactor diodes, a pair of open-ended coupled lines and a U-shape step impedance line (SIL) with an open-ended stub loaded at the centre of the SIL to form a very compact layout. The odd- and evenmode analysis and equivalent circuit model are demonstrated for estimation of the transmission characteristics. Tuning the DC voltage applied on the varactor diodes, the varactor capacitance accordingly changes leading to a varying cutoff frequency $f_{\mathrm{c}}$. The measured results show that the achieved $3-\mathrm{dB} f_{\mathrm{c}}$ tuning range is $60.6 \%$ (1.15 to 2.15 $\mathrm{GHz}$ ). The measured insertion loss (IL) and roll-off rate are $0.2-0.4 \mathrm{~dB}$ and $50-73 \mathrm{~dB} / \mathrm{GHz}$, respectively. The overall size of the LPF is only $0.005 \lambda_{\mathrm{g}}{ }^{2}$, which shows a competitive advantage comparing with the state-of-the-art work.

Introduction: As one of the core components in wireless communication systems, lowpass filters (LPF) with sharp roll-off frequency response, low insertion loss and compact size play an important role in down-conversion link of the receivers [1]. Recently, the frequency-tunable LPF designs have been comprehensively studied using various technologies, such as defected ground structure (DGS) [2] and complementary split ring resonator-split ring resonator (CSRRSRR) [3]. It is noted that the tunable LPF is highly desired in the reconfigurable wireless communication systems. Huang et al. presented a coplanar waveguide (CPW) tunable lowpass filter with the tuning range of $49 \%$ and a size of $0.2 \times 0.318 \lambda_{\mathrm{g}}{ }^{2}$ [4]. Ni and Hong proposed a continuously tunable microstrip LPF with a sharp roll-off [5], however, it has a complicated tuning mechanism using five tuning devices and two different DC control-voltages leading to a deteriorated insertion loss. In [6], a tunable low-pass filter using variable mode impedance of coupled structure is proposed. Even though the concept is quite new, the deteriorated insertion loss is inherently challenging to be improved. The tunable LPF designed by Cai et al. [7] has a compact size and ultrawide stopband, however the achieved roll-off over the tuning range is significantly degraded to $16-33 \mathrm{~dB} / \mathrm{GHz}$. Therefore, an optimized tunable LPF compromising the above-mentioned challenges is highly desired in the emerging reconfigurable communication systems.

In this paper, a compact LPF with low insertion loss, sharp roll-off and wide tuning range is realized using a very simple structure. To prove the concept, the proposed LPF without tuning components is presented in Fig. 1a. Both the odd- and even-mode and lumped equivalent circuit models can be derived for the LPF design according to the layout of the proposed LPF. To create a tunable LPF, two varactor diodes are connected at the end of the optimized center-loaded stub as is shown in Fig. $1 b$. The measured results verify that the proposed tunable LPF has the lowest insertion loss, compact size and very competitive performance in terms of tuning range and roll-off.

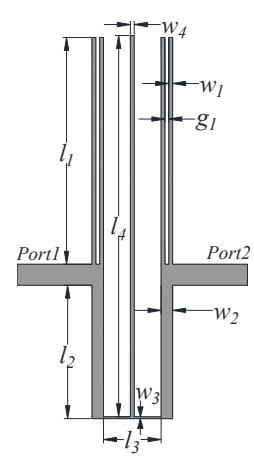

$a$

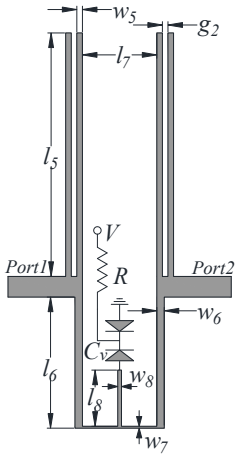

Fig. 1 Layout of the proposed lowpass filter.

$a$ Without varactor

$b$ With varactors loaded on open-ended stub
Proposed LPF: the lossless transmission-line circuit model of the proposed LPF without tuning components is shown in Fig. $2 a$, which is formed by a pair of open-ended coupled line and a U-shape step impedance line (SIL) with the open-ended stub loaded at the center of the SIL. In Fig. $2 a, \theta_{\mathrm{i}}(\mathrm{i}=1,2,3,4)$ signifies the electrical length and $\mathrm{Y}_{\mathrm{j}}$ $(j=2,3)$ is the characteristic admittance of each section. $Y_{o o} / Y_{o e}$ are the odd/even characteristic admittances of the coupled lines. Fig. $2 b$ presents a lumped equivalent circuit model of the LPF. The pairs $\mathrm{L}_{1} / \mathrm{C}_{1}$ and $\mathrm{L}_{2} / \mathrm{C}_{2}$ correspond to the open-ended coupled lines and the step impedance line, $\mathrm{C}_{3}$ represents the open-ended stub taped on the center of the SIL. Fig. 3 shows the odd- and even-mode circuit models of proposed LPF. The input admittance $\mathrm{Y}_{\text {ino }}$ and $\mathrm{Y}_{\text {ine }}$ can be deduced as

$$
\begin{gathered}
Y_{\text {ino }}=j 2 Y_{\text {oo }} \tan \theta_{1}+Y_{2} \frac{j\left(Y_{2} \tan \theta_{2}-Y_{3} \cot \theta_{3}\right)}{Y_{2}+Y_{3} \cot \theta_{3} \tan \theta_{2}} \\
Y_{\text {ine }}=j 2 Y_{\text {oo }} \tan \theta_{1}+Y_{2} \frac{j\left(Y_{2} \tan \theta_{2}+Y_{3} \tan \left(\theta_{3}+\theta_{4}\right)\right)}{Y_{2}-Y_{3} \tan \left(\theta_{3}+\theta_{4}\right) \tan \theta_{2}}
\end{gathered}
$$

Accordingly, the S-parameters of the LPF can be expressed as

$$
S_{11}=\frac{Y_{o}^{2}-Y_{\text {ino }} Y_{\text {ine }}}{\left(Y_{o}+Y_{\text {ino }}\right)\left(Y_{o}+Y_{\text {ine }}\right)}, S_{21}=\frac{Y_{o}\left(Y_{\text {ino }}-Y_{\text {ine }}\right)}{\left(Y_{o}+Y_{\text {ino }}\right)\left(Y_{o}+Y_{\text {ine }}\right)}
$$

where, $Y_{0}$ is the port admittance. So equations (1)-(3) can be used to depict the transmission characteristics and predict the transmission zeros and poles during LPF design.

The equivalent circuit models in Fig. 2 and 3 are applied for LPF design and analysis. The frequency responses using equation-based calculation (3), EM simulation and LC-equivalent circuit simulation are compared in Fig. $4 a$, where the substrate RT4350 ( $\varepsilon_{r}=3.66, h=0.508$ $\mathrm{mm})$ is applied. The physical dimensions of the proposed LPF are: $l_{l}=11.9 \mathrm{~mm}, l_{2}=7 \mathrm{~mm}, l_{3}=3 \mathrm{~mm}, l_{4}=20 \mathrm{~mm}, w_{l}=0.2 \mathrm{~mm}, w_{2}=0.6 \mathrm{~mm}$, $w_{3}=0.1 \mathrm{~mm}, w_{4}=0.2 \mathrm{~mm}, g_{l}=0.2 \mathrm{~mm}$. The curve-fitted equivalent circuit model parameters are: $\mathrm{C}_{1}=1.7 \mathrm{pF}, \mathrm{C}_{2}=1.4 \mathrm{pF}, \mathrm{C}_{3}=2 \mathrm{pF}, \mathrm{L}_{1}=2.8 \mathrm{nH}$, $\mathrm{L}_{2}=2.6 \mathrm{nH}$. It can be seen from Fig. $4 a$ that the frequency responses have a good agreement among the three approaches verifying an accurate design methodology of the proposed LPF.

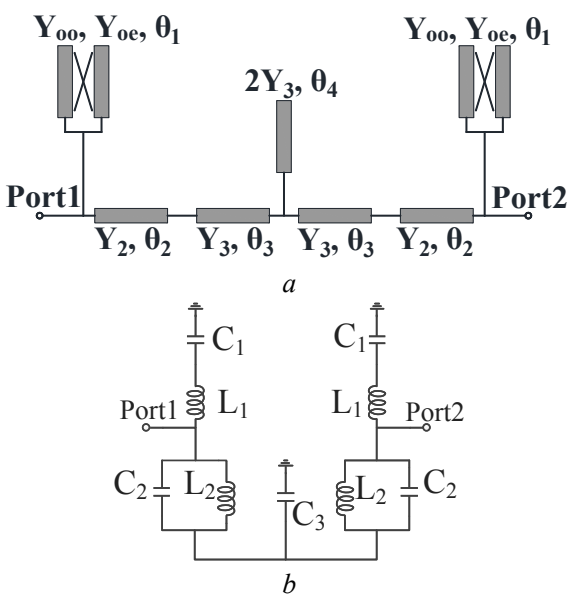

Fig.2 Circuit models of the proposed lowpass filter. $a$ Lossless transmission line model $b$ LC-equivalent circuit model

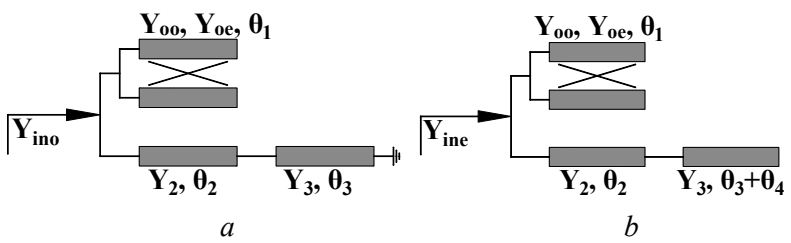

Fig. 3 Odd-and even-mode models of proposed filter. $a$ Odd-mode circuit model $b$ Even-mode circuit model 


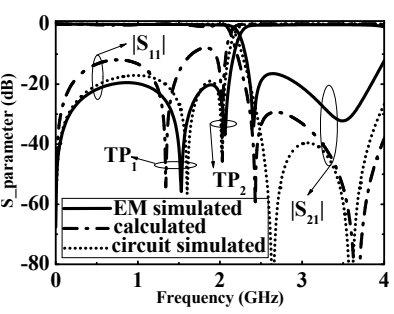

$a$

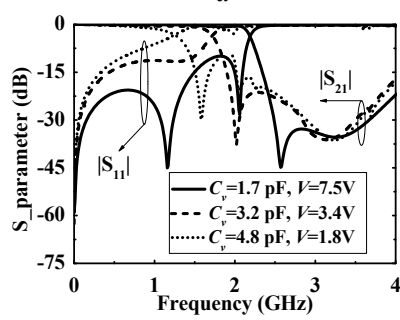

$c$
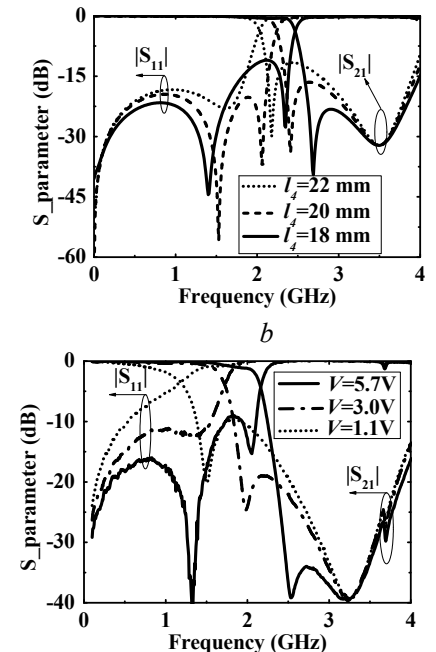

$d$
Fig. 4 Frequency responses of the proposed tunable LPF. $a$ Equation-based calculation, EM simulation and LC-circuit simulation $b$ Simulated results with different length $l_{4}$

$c$ Simulated results with different capacitor $C_{v}$

$d$ Measured results with different tuning voltage

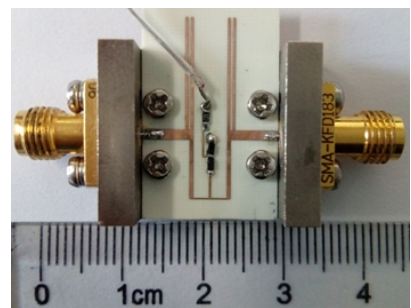

Fig. 5 Photograph of the fabricated tunable LPF.

The two transmission poles (TP1 and TP2) as shown in Fig. $4 a$ are determined by the odd- mode and even- mode resonant frequencies, which are given by equation (3).

To explore the tuning mechanism of this $\mathrm{LPF}$, the variation of $3-\mathrm{dB}$ cutoff frequency $f_{\mathrm{c}}$ against $l_{4}$ is shown in Fig. $4 b$, where $l_{4}$ is corresponding to the capacitive impedance $\mathrm{C}_{3}$ as shown in Fig. $2 b$. According to Fig. $4 b$, as the length $l_{4}$ increases from 18 to $22 \mathrm{~mm}, f_{\mathrm{c}}$ and TP2 move towards the lower frequencies while TP1 almost remains unchanged. This is because the length $l_{4}$ only affects even mode frequency according to equations (1) and (2).

Based on the above analysis, two varactor diodes are placed at the end of the open-ended stub taped on the center of the U-shape SIL. The two varactor diodes are corresponding to the varying capacitance indicated by the length $l_{4}$. The layout of the proposed tunable LPF is shown in Fig. $1 b$. After optimization, the dimensions of the proposed tunable LPF are: $l_{5}=13 \mathrm{~mm}, l_{6}=7 \mathrm{~mm}, l_{7}=4 \mathrm{~mm}, l_{8}=3 \mathrm{~mm}, w_{5}=0.3 \mathrm{~mm}, w_{6}=0.4 \mathrm{~mm}$, $w_{7}=0.1 \mathrm{~mm}, w_{8}=0.2 \mathrm{~mm}, g_{2}=0.3 \mathrm{~mm}$. The junction capacitance of the diodes SMV-1234-079LF changes from 1.7 to $4.8 \mathrm{pF}$. The bias resistor of $270 \Omega$ is applied to block the RF signal leakage. Fig. $4 c$ shows the simulated frequency responses of the proposed tunable LPF with different capacitor $C_{v}$. It is noted that, when the $C_{v}$ increases, the cutoff frequency $f_{\mathrm{c}}$ tends to shift downward with a reduced upper-stopband attenuation. The roll-off remains within a relatively stable range varying between 60 and $77 \mathrm{~dB} / \mathrm{GHz}$.

Experiment and discussion: The proposed tunable LPF was fabricated and measured by a R\&S ZVL6 vector network analyzer. Fig. 5 shows the photograph of proposed tunable LPF. In Fig. $4 d$, three groups of the measured frequency responses are demonstrated. As is shown, when the voltage is $1.1,3$ and $5.7 \mathrm{~V}$, respectively, the $3-\mathrm{dB}$ cutoff frequency is tuned to $1.15,1.7,2.15 \mathrm{GHz}$, respectively, while maintaining a return loss better than $10 \mathrm{~dB}$. What's more, the measured roll-off varies between 50 to $73 \mathrm{~dB} / \mathrm{GHz}$. The difference between the simulated (Fig.
$4 c$ ) and measured (Fig. $4 d$ ) results owes to fabrication toleration and accuracy of the diode SPICE model.

Table 1 shows a comparison between the proposed tunable LPF and other state-of-the-art work. It is obvious that the proposed tunable LPF is not only realized in a simple layout pattern, but also presents a very competitive performance over the counterparts.

Table 1: Comparisons with state-of-the-art tunable LPFs

\begin{tabular}{|c|c|c|c|c|}
\hline Ref. & $\begin{array}{c}3-\mathrm{dB} f_{\mathrm{c}} \text { tuning } \\
\text { range }(\mathrm{GHz})\end{array}$ & $\mathrm{IL}(\mathrm{dB})$ & $\begin{array}{c}\text { Roll-off } \\
(\mathrm{dB} / \mathrm{GHz})\end{array}$ & $\begin{array}{c}\text { Size } \\
\lambda_{\mathrm{g}}{ }^{2}\end{array}$ \\
\hline 4 & $49 \%(1.29-2.13)$ & 0.7 & $24-30$ & 0.064 \\
\hline 5 & $59 \%(1.6-2.94)$ & $\begin{array}{c}0.3- \\
0.8\end{array}$ & $48-55$ & 0.008 \\
\hline 6 & $50 \%(1.5-2.5)$ & 1 & $43-85$ & 0.027 \\
\hline 7 & $91.3 \%(0.85-2.28)$ & 0.8 & $16-33$ & 0.007 \\
\hline $\begin{array}{c}\text { This } \\
\text { work }\end{array}$ & $60.6 \%(1.15-2.15)$ & $\begin{array}{c}0.2- \\
0.4\end{array}$ & $50-73$ & 0.005 \\
\hline
\end{tabular}

* Roll-off : $\mid \mathrm{a}_{\max }-\mathrm{a}_{\min } / / f s$ - $f t \mid$ where $\mathrm{a}_{\max }$ is $20 \mathrm{~dB}$ attenuation, $\mathrm{a}_{\min }$ is $3 \mathrm{~dB}$ attenuation, $f s$ is the frequency corresponding to $20 \mathrm{~dB}$ attenuation and $f t$ is the frequency corresponding to $3 \mathrm{~dB}$ attenuation; $\lambda_{\mathrm{g}}$ is the guided wavelength at the lowest $f_{c}$.

Conclusion: A novel compact tunable lowpass filter with low insertion loss, sharp roll-off and wide tuning range realized in a simple structure is reported in this paper. The experimental results approve that the proposed design has the merits of low insertion loss (0.2-0.4 dB), sharp roll-off $(50-73 \mathrm{~dB} / \mathrm{GHz})$ and wide tuning range $(60.6 \%)$. The miniaturized design only has a size of $0.005 \lambda_{\mathrm{g}}{ }^{2}$ which is a cost-effective and easy-to-integrate solution in the reconfigurable wireless systems.

Acknowledgments: This work was supported by the National Natural Science Foundation of China (U1430102) and the Fund of key laboratory (KF14005).

Ting Zhang, Zongqi Cai, Jingfu Bao (School of Electronic Engineering, University of Electronic Science and Technology of China, Chengdu, 611731, China)

Yang Yang (Global Big Data Technology Centre, University of Technology Sydney, Sydney, NSW 2007, Australia)

E-mail: yang.yang.au@ieee.org

Yiwen Wang (School of Microelectronics and Solid-State electronics, University of Electronic Science and Technology of China, Chengdu, 611731, China)

\section{References}

1 Hong. J,: 'Reconfigurable planar filters', IEEE Microwave Magazine, 2009, 10, (6), pp. 73-83.

2 Kufa, M. and Raida, Z.: 'Lowpass filter with reduced fractal defected ground structure', Electron. Lett., 2013, 49, (3), pp. 199-201.

3 Yang, Y., Zhu, X., Karmakar, N.: 'Microstrip lowpass filter based on split ring and complementary split ring resonators', Microw. Opt. Technol. Lett., 2012, 54, (7), pp. 1723-1726.

4 Huang. C. C, Chen. N. W, Tsai. H. J, et al,: 'A coplanar waveguide bandwidth-tunable lowpass filter with broadband rejection', IEEE Microw. Wirel. Compon. Lett, 2013, 23, (3), pp. 134-136.

$5 \mathrm{Ni}$. J, Hong. J,: 'Compact continuously tunable microstrip low-pass filter', IEEE Trans. Microw. Theory Tech, 2013, 61, (5), pp. 17931800.

6 Abbosh. A.: 'Compact tunable low-pass filter using variable mode impedance of coupled structure', IET Micro., Ant. \& Propag., 2012, 6, (12), pp. 1306-1310.

7 Cai. C, Wang. J, Zhang. G.: Tunable microstrip lowpass filter with compact size and ultra-wide stopband, Electron. Lett., 2015, 51, (19), pp. 1514-1516. 